\title{
Train Timetable Stability Evaluation based on Analysis of Interior and Exterior Factors Information Entropy
}

\author{
Xuelei Meng ${ }^{1,2, *}$ and Limin Jia $^{2, *}$ \\ ${ }^{1}$ School of Traffic and Transportation, Lanzhou Jiaotong University, Lanzhou 730070, P. R. China \\ ${ }^{2}$ State Key Laboratory of Rail Traffic Control and Safety, Beijing Jiaotong University, Beijing 100044, P. R. China
}

Received: 19 Jun. 2013, Revised: 24 Oct. 2013, Accepted: 25 Oct. 2013

Published online: 1 May. 2014

\begin{abstract}
To evaluate the quality of the train timetable roundly, train timetable stability is defined based on the analysis of the interior and exterior factors affecting the timetable stability. The exterior factors include the grade of the railway lines, the blocking system type, the station interlocking type, the leading locomotive performance and the average mileage of the sections. The interior factors are the capacity load of the sections, the capacity load of the stations, the total time supplement for running in the sections, the total time supplement for dwelling on the stations, the timetable periodic time and the buffer times between train paths. We define the information entropy of the exterior factors and the interior factors and present the equation to calculate the information entropy. Then train timetable stability is quantized according to the definitions above. The computing case proves the method to evaluate the train timetable stability is feasible and efficient.
\end{abstract}

Keywords: Train timetable, stability, exterior factor, interior factor, information entropy

\section{Introduction}

Train timetable is the key file for organizing the railway traffic, which determines the inbound and outbound time of trains. Railways typically operate according to a planned (predetermined) timetable, and the quality of the timetable determines the quality of the railway service. So mapping out a high quality timetable for diverse trains is rather important.

Therefore, to ensure both the capacity and the order of the train operation, it is necessary to work out a reliable, stable, robust timetable and the feasible efficient re-scheduling of the planned timetable. The reliability of a timetable is the capability to finish all the trains' trips within the given time, no matter what happens. The stability of a timetable is the availability that the timetable can afford for the trains to recover the situation that they run as the timetable has stipulated after the trains are disturbed when running. The robustness of a timetable is the capability of a timetable to counteract the disturbances and remain its original shape as planned.

A high quality timetable not only can decide the inbound and outbound time at stations, and more importantly, it offers the possibility to recover the operation according to the planned timetable when the trains are disturbed by accidents randomly. Timetable stability is the index to measure this possibility. Timetable stability is closely related to the train number assigned to the railway sections and the time supplement distributed to each station and section, the probability that the train is disrupted at the stations and in the sections. So while assigning the trains paths and mapping out the train schedule, we should deliberately design the train number assigned to the sections and time supplement distribution, not only considering the section capacity and station capacity, but also the minimum running time at each section and the minimum dwell on the station. Section capacity is the maximum number of trains that can run over the section within a certain period of time. Station capacity is the maximum number of trains that the station can receive and send within a period of time.

Timetable stability evaluation is a relatively new issue in the field of railway operation research. The research work was first published in the 1990s, beginning with the dynamic indexes of the timetable [1]. The dynamic indexes also included the adjustability [2-4], equilibrium $[4,5]$ and the rescheduling elasticity [6]. The indexes were based on the more fundamental factors: the

\footnotetext{
*Corresponding author e-mail: researches@126.com, jialm@ vip.sina.com
} 
utilization coefficient of the section capacity and the time supplement in the sections and on the stations [3].

The research experienced two stages. In the initial period, it focused on the timetable of the railway dispatching section. Yang pointed out the basic rules of appraising the vibrant performance of the train operation diagram from delay spread simulation and set up the corresponding dynamic index system [1]. Peng et al analyzed the influences of time supplement and its distribution pattern on train delay propagation in a given diagram and built the timetable elasticity evaluating system [3]. And they proposed the method to evaluate the timetable equilibrium [5]. They formed the basis to study the train timetable stability. Goverde built a discrete dynamic system model to describe the timetable with the max-plus algebra based on the discussion of the timetable periodicity and analyzed the timetable stability [7]. Carey developed a simulation model to test the schedule performance and reliability for train stations [8]. Hansen pointed out that the effect of the stochastic disturbance on trains relied on the adjustment of the running time and buffer time in the timetable. He also analyzed the advantages and disadvantages of the capacity and stability evaluating model [9]. Zha et al used interval estimation of the random variable under the specified level of significance in mathematics statistics, and analyzed the time standard of the train running time in the section and effects of the cushion time of train operation diagram [10]. These researchers promoted the train timetable stability theory from the perspective of the running time in railway sections, the dwelling time on railway stations and the buffer time between train paths. De Kort et al proposed a method to evaluate the capacity determined by the timetable and took the timetable stability as a part of the capacity. The goal was to place train running paths as much as possible, while taking the timetable into consideration at the same time [11]. Goverde presented the method based on max-plus to analyze the timetable stability and took the Netherlands national railway timetable as a computing case to prove the feasibility of using the method to design the timetable of the railway network [12]. Zong and $\mathrm{Li}$ studied the reasonable time supplement of the trains running in the sections [13]. Chen introduced the timed-PETRI network to build the trains operation model and utilized Exspect(a simulation software) to simulate the model and evaluate the timetable stability [14]. We defined and qualified timetable stability, and took it as a goal when rescheduling trains on the dispatching sections [15].

So it is clear that time is the key factor to study the timetable stability of a dispatching railway section. Focusing on the delay time [1], the behind schedule ratio $[1,3]$, the time supplement [9, 13], time deviation [5], researchers studied the timetable adjustability, equilibrium, stability with theories of statistics, max-plus algebra, etc.

In the following period, there is an increasing move toward networked timetable stability evaluation because the previous timetable stability evaluation based on dispatching section doesn't suit the networked timetable design and optimization. Engelhardt-Funke and Kolonko considered a network of periodically running railway lines. They built a model to analyze stability and investments in railway networks and designed an advanced evolutionary algorithm to solve the problem [16]. Goverde analyzed the dependence of the timetable on the busy degree of the railway network. He again hired the max-plus algebra to analyze the timetable stability of the railway network. On this basis, he proposed a new method to generate the paths for the trains on a large-scale railway network [17]. Delorme et al presented a station capacity evaluating model and evaluated the stability on the fundamental parts of the railway network: stations [18]. We analyzed the complex characteristic analysis of passenger train flow network [19] and studied the train flow vulnerability in the previous study [20].

And we can see that the timetable stability is not only related to time but also the utilization coefficient capacity of the railway network $[17,18]$. That is to say, the networked timetable stability study requires the combination of the railway network capacity utilization and the time supplement distribution in the sections and on the stations. Most of the publications are concerned with the stability of the timetable for a single dispatching railway section, whereas, only a few publications are about the networked timetable stability. Furthermore, the research on the timetable is in the stage of evaluating, mostly qualitative, not the quantization of the timetable stability.

The essence of the timetable stability improvement is to utilize the transport resources more efficiently and maximize the function of the railway transportation system. It is the transportation resources in the railway transportation system that corresponds with the thermal energy of a thermodynamic system. Then the entropy is used to describe the distribution situation of the transportation resources, such as the time supplement, buffer time, in railway transportation system. On the one hand, compared with the distribution of thermal energy in a thermodynamic system, the more symmetrically the transportation resources are distributed, the more chaotic the situation will be. Then the timetable is less stable. On the other hand, although the transportation resources are sufficient, the resources will be wasted without well-designed distribution plan, which may lead to weak stability of the networked timetable. So we can conduct a research into timetable stability evaluation based on entropy theory.

We first quantize timetable stability index. Then we analyze the factors affecting timetable stability, including exterior factors and interior factors and hire information entropy theory to describe the timetable stability indices. We proposed an innovated method to evaluate the timetable stability. 
The outline of the paper is as follows. Section 1 gives the introduction on the timetable stability evaluating problem. Section 2 analyzes the interior and exterior factors affecting the train timetable stability. Then Section 3 defines the train timetable stability based on the information entropy of the interior and exterior factors. The computing case is presented in Section 4. Finally, Section 5 gives some conclusions.

\section{Analysis of factors affecting timetable stability}

\subsection{Definition of train timetable stability}

In reference [21], the timetable stability is described from three aspects:

-Conflict stability: A timetable is called conflict stable, if the expected number of conflicts does not exceed a desired maximal number of conflicts, assuming that the train delay distributions are given.

-Structural stability: A timetable is called structurally stable, if the maximum probability that two routes will become incompatible does not exceed a desired maximal probability, assuming that the train delay distributions are given.

-Cluster stability: A timetable is called cluster stable, if the expected number of conflicts of each train does not exceed a desired maximal number of conflicts per train, assuming that the train delay distributions are given.

In reference [22], the stability is defined as the system sensitivity and recovering ability under the threshold on the assumption that the train contrail deviates as planned. We can see that the timetable stability is also defined based on the number of conflicts and a threshold setting for comparison. And the number of conflicts is obviously determined by the train number and the section transport capacity and the station capacity. Therefore, it is important to take the capacity of the sections and stations and the distributing plan of the trains into account while defining the timetable stability. They are deep-seated tangible factors to affect the timetable stability. Thus, we define timetable stability as follows.

Definition 1: Train timetable stability is the ability provided by the timetable to recover the train operation status according to the planned timetable when disturbed by the emergencies in a condition of a railway network with a certain capacity.

According to the definition, we must consider not only the exterior factors, such as the section transport capacity and the station approaching and sending train capacity, but also the interior factors which are equally important in affecting the timetable stability.

\subsection{Exterior factors of the train timetable stability}

We consider the railway network as a frame, which accommodates the railway traffic. The hardness of the frame represents the capacity to endure the traffic volume, which is related to the exterior factors affecting timetable stability. The exterior factors to decide the networked train timetable stability are as follows.

(1) The grade of the railway lines

The grade of the railway lines is a symbol of the line capacity. The line grade determines the maximal curve on the line, the train running speed, the minimum tracking interval time between two trains, even the block system. These are all the factors to affect the capability of the railway network. The higher grade the railway belongs to, the bigger transport capacity it has, which means the higher stability for the timetable.

(2) The blocking system type

There are mainly three kinds of blocking system today: semiautomatic block system, automatic block system and moving block system. Different block systems allow different minimum tracking interval time, which enormously affects the capacity of the railway. Among three of them, the moving block system produces the largest capacity, benefiting the networked timetable stability in the farthest way.

(3)The station interlocking type

Today electric interlocking and computer interlocking systems are all in use. The response speed of the interlocking system is the key factor to affect the station capacity of receiving and sending out trains. The deeper level reasons include the communication capability, the computing ability, the reliability and the fault-tolerant ability.

Compared with the electric interlocking system, the computer interlocking system can enhance the station capacity better, accordingly, the latter can produce higher timetable stability than the former.

(4) The leading locomotive performance

The traction of the leading locomotive determines the train speed to a certain extent, and then affects the running time at the railway sections. So the leading locomotive performance is also a factor affecting the section capability.

(5) The average mileage of the sections

The mileage of the sections can affect the section transport capability to a certain extent, especially on the single-track railway. Mileage can affect the running time of trains in the sections, which will affect the timetable stability.

\subsection{Interior factors of the train timetable stability}

The spatiotemporal relationship between the trains and the two components of railway, sections and stations, 
determined by the timetable decides the capacity load of each part of the railway. The capacity load is the key interior element affecting the timetable.

(1)The capacity load of the sections

The capacity load of each section is the ratio of the train number running through the section according to the timetable for a day to the section capacity. If the capacity load is heavy, it is very difficult to reschedule the trains when they are disturbed and the hope to recover the status that the trains are running according to the planned timetable becomes slim. The bigger the capacity load is, the smaller the timetable stability value becomes.

(2)The capacity load of the stations

The capacity load of each section is the ratio of the train number arriving at and departing from the station according to the timetable for a day to the station capacity for receiving and sending trains. Similar to the capacity load of the sections, if the capacity load is heavy, it is very difficult to reschedule the trains when the trains are disturbed and we have slim hopes to recover the status that the trains are running according to the planned timetable. The bigger the capacity load is, the smaller the timetable stability value is.

(3) The total time supplement for running in the sections

The total time supplement for running in the sections is the summation of the running time supplement for every train in all the sections. The time supplement reserved for the train running can absorb some slight delay of the trains, coping with the disturbances in the process of trains running. The more time supplement is reserved, the more stable the train timetable will be.

(4) The total time supplements for dwelling on the stations

Similar to the total time supplement for running in the sections, the more time supplement for trains dwelling on the stations, the more stable the timetable will be.

(5) The timetable periodic time

The planned running trajectories of the trains are lined in the timetable graph circularly, which is called a path. The time that a path occupies is called a train graph cycle. On the single-track railway, the train graph cycle is the factor determining the capacity of the timetable. The shorter the train graph cycle is, the more stable the train timetable will be.

(6) The buffer times between train paths

The buffer time between two train paths is the allowance time that is added to the minimum time between two train paths. It is well known that there must be a period of time between two train paths, called minimum tracing time, which can ensure the safety of the trains running in the sections under a certain type of blocking system. Buffer times must be added on the minimum time between train paths to prevent or reduce delay propagation. So we can see that the more buffer time exists between train paths, the more stable a timetable will be.

\section{Timetable stability}

\subsection{Information entropy}

Shannon pointed out that redundancies occur in all kinds of information. The size of the redundancy is related to the ratio of each character or the uncertainty [23]. He proposed the definition of information entropy and the equation calculating the entropy. If $p_{i}$ is the probability of a specified signal, the summary information of several signals is $-\sum p_{i} \ln p_{i}$. Then $H=-c \sum p_{i} \ln p_{i}$ is defined as the information entropy, where $c$ is a proportionality constant. Thermodynamics is increasingly used in transportation problem modeling, showing its availability. Bianca and Coscia hired the kinetic theory (which is also a branch of thermodynamics) to deal with the derivation and the analysis of a new mathematical model for vehicular traffic along a one-way road obtained by the coupling of a uniform and an adaptive discretization of the velocity variable [24]. In this paper, we hire entropy theory, which is also a branch of thermodynamics to study the timetable stability evaluating problem.

\subsection{Timetable information entropy}

$e_{i}$ is assumed to be the factor affecting timetable stability, $i=1,2, \cdots, N$. The interacting determinants of factor $k$ on factor $j$ is $f_{k j}, k=1,2, \cdots, N, j=1,2, \cdots, N$. Then the interacting determinants form a matrix $F$. The affecting index number of factor $e_{i}$ is $p_{i}$, then

$$
p_{i}=\frac{\sum_{k=1}^{N} f_{k i}+\sum_{j=1}^{N} f_{i j}}{\sum_{i=1}^{N}\left(\sum_{k=1}^{N} f_{k i}+\sum_{j=1}^{N} f_{i j}\right)}=\frac{\sum_{k=1}^{N} f_{k i}+\sum_{j=1}^{N} f_{i j}}{2 \sum_{k=1}^{N} \sum_{j=1}^{N} f_{k j}}
$$

In Equation (1), the numerator is the summary of the interacting determinants of factor $e_{i}$ acting on other determinants and received from other determinants. The denominator is the summary of all the interacting determinants between the factors.

Then the information entropy of train timetable stability is

$$
S=-c \sum p_{i} \ln p_{i}
$$

where $c=1 / \ln (N)$, which is the proportionality constant to define the timetable stability. $N$ is the number of the factors which affect the timetable stability.

\subsection{Normalization of timetable stability information entropy}

The timetable stability entropy is the key index to evaluate the train timetable stability. When the timetable is stable, the factors are balanced and then the information 
entropy is high. When the factors present an ordered appearance, the information entropy is low. But when the information entropy is used to evaluate the timetable stability, the problem is that it does not have a definitive value range, which makes it rather difficult to analyze the timetable stability with the information entropy.

In the previous publication, such as Herrmann's dissertation, timetable stability research is on the qualitative level and there is no clearly defined boundary to tell the timetable stability [21]. It is quite difficult to define the required number in the definition and it is very hard to tell whether the timetable is stable. Timetable stability has the typical fuzzy characteristics and we hired the fuzzy theory to describe it in a quantitative manner. Triangle function is the most commonly used function to describe such characteristics and it is utilized in this paper. So the information entropy can be normalized by the membership degree functions, as follows.

$$
G=q(S), 0 \leq q(S) \leq 1
$$

The triangle function is selected to map the relationship from timetable stability entropy to timetable stability index as follows.

$$
G= \begin{cases}0 & S \leq M \\ \frac{S-M}{L-M} & M<S<L \\ 1 & S \geq L\end{cases}
$$

$M$ is the lower bound of $S$ and $L$ is the upper bound of $S$. Of course, other membership degree functions can be used to define $q$, such as the trapezoidal function, Sigmoid function and Gauss function.

\subsection{Timetable stability value}

The timetable stability is affected by two kinds of factors, the interior factors and the exterior factors. So the information entropy can be divided into two kinds. One is the interior factor timetable stability index $G^{\text {interior }}$, the other is exterior factor timetable stability $G^{\text {exterior }}$. Then the timetable stability value is defined as follows.

$$
W=G^{\text {interior }} \times G^{\text {exterior }}
$$

\section{A computing case}

We took the timetable of Beijing-Guangzhou high speed railway, section from Beijing to Zhengzhou and from 8:00 to 12:00 as the evaluating target, shown in Fig. 1. It is equipped with moving block system and computer interlocking system. The railway line has two tracks.

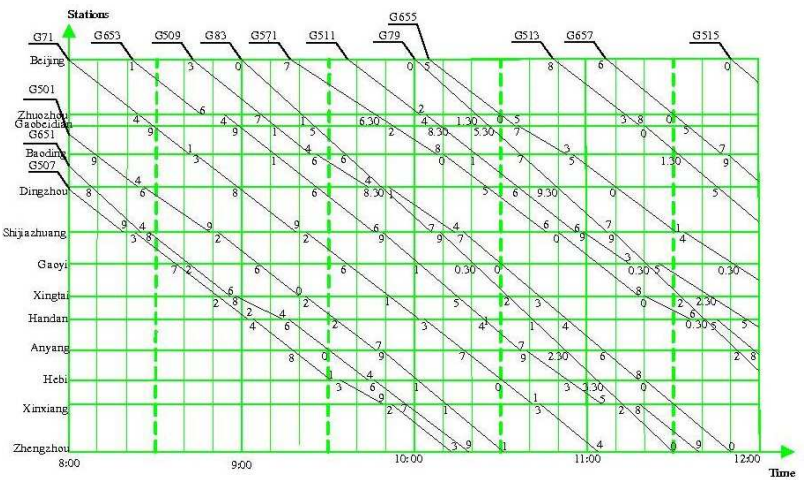

Fig. 1: The timetable from Beijing to Zhengzhou from 8:00 to 12:00

\subsection{The exterior timetable stability index}

$e_{1} \sim e_{5}$ represent the grade of the railway lines, the blocking system type, the station interlocking type, the leading locomotive performance and the average mileage of the sections respectively. The interacting determinants between these five exterior factors are shown in Table 1.

Table 1: Interacting determinants between five exterior factors

\begin{tabular}{ccccccc}
\hline & $e_{1}$ & $e_{2}$ & $e_{3}$ & $e_{4}$ & $e_{5}$ & Total \\
\hline$e_{1}$ & - & 5 & 5 & 3 & 5 & 18 \\
$e_{2}$ & 2 & - & 4 & 3 & 2 & 11 \\
$e_{3}$ & 1 & 4 & - & 2 & 4 & 10 \\
$e_{4}$ & 0 & 2 & 5 & - & 3 & 10 \\
$e_{5}$ & 1 & 2 & 5 & 4 & - & 12 \\
Summary & 4 & 13 & 19 & 12 & 14 & 62 \\
$p_{i}$ & 0.177 & 0.194 & 0.242 & 0.177 & 0.210 & \\
$\ln \left(p_{i}\right)$ & -1.729 & -1.642 & -1.419 & -1.729 & -1.562 & \\
\hline
\end{tabular}

According to the algorithm in Section 2.2, the proportionality constant and the exterior information entropy are calculated as follows.

$$
\begin{gathered}
c=1 / \ln (5)=0.621 \\
S^{\text {exterior }}=-c \sum p_{i} \ln p_{i}=-0.621[0.177 \times(-1.729) \\
+0.194 \times(-1.642)+0.242 \times(-1.419) \\
+0.177 \times(-1.729)+0.210 \times(-1.562)]=0.996
\end{gathered}
$$

According to Equation (4), the exterior timetable stability index calculating function is set to be

$$
G^{\text {exterior }}= \begin{cases}0 & S^{\text {exterior }} \leq 0.9 \\ \frac{\text { Sexterior }_{-0.9}}{1-0.9} & 0.9<S^{\text {exterior }}<1 \\ 1 & S^{\text {exterior }} \geq 1\end{cases}
$$

where $M$ is set to be 0.9 . The value is also obtained from the questionnaires sent back by the researchers and experts. 
Then the exterior timetable stability index is $G^{\text {exterior }}=$ $\frac{0.996-0.9}{1-0.9}=0.96$.

\subsection{The interior timetable stability index}

$h_{1} \sim h_{6}$ stand for the capacity load of the sections, the capacity load of the stations, the total time supplements for running in the sections, the time supplements for dwelling on the stations, the timetable periodic time and the buffer time between train paths respectively. The interacting determinants among these six interior factors are shown in Table 2.

Table 2: Interacting determinants between five interior factors

\begin{tabular}{cccccccc}
\hline & $h_{1}$ & $h_{2}$ & $h_{3}$ & $h_{4}$ & $h_{5}$ & $h_{6}$ & Total \\
\hline$h_{1}$ & - & 5 & 5 & 4 & 2 & 4 & 20 \\
$h_{2}$ & 5 & - & 4 & 0 & 4 & 2 & 15 \\
$h_{3}$ & 3 & 3 & - & 4 & 1 & 3 & 14 \\
$h_{4}$ & 3 & 3 & 4 & - & 3 & 4 & 17 \\
$h_{5}$ & 5 & 4 & 1 & 2 & 0 & 3 & 15 \\
$h_{6}$ & 4 & 2 & 3 & 5 & 4 & 0 & 18 \\
Summary & 20 & 17 & 17 & 15 & 14 & 16 & 99 \\
$p_{i}$ & 0.202 & 0.162 & 0.157 & 0.162 & 0.146 & 0.172 & \\
$\ln \left(p_{i}\right)$ & -1.599 & -2.344 & -1.854 & -1.823 & -1.921 & -1.762 & \\
\hline \multicolumn{7}{c}{} & \multicolumn{7}{c}{}
\end{tabular}

$$
\begin{gathered}
c=1 / \ln (6)=0.558 \\
S^{\text {exterior }}=-c \sum p_{i} \ln p_{i}=-0.558[0.202 \times(-1.599) \\
+0.162 \times(-2.344)+0.157 \times(-1.854) \\
+0.162 \times(-1.823)+0.146 \times(-1.921) \\
+0.172 \times(-1.762)]=0.997
\end{gathered}
$$

According to Equation (4), the interior timetable stability index calculating function is set to be

$$
G^{\text {interior }}= \begin{cases}0 & S^{\text {interior }} \leq 0.9 \\ \frac{S_{\text {interior }}-0.9}{1-0.9} & 0.9<S^{\text {interior }}<1 \\ 1 & S^{\text {interior }} \geq 1\end{cases}
$$

Then the interior timetable stability index is $G^{\text {interior }}=$ $\frac{0.997-0.9}{1-0.9}=0.97$.

At last, according to definition of the timetable, shown in Equation (5), the timetable stability value is $W=G^{\text {interior }} \times G^{\text {exterior }}=0.96 \times 0.97=0.9312$.

\subsection{Analysis of the computing results}

It can be seen from Section 4.1 and Section 4.2 that the computing results totally depend on the interacting determinants among the affecting factors. Values of interacting determinants among the factors are the superficial elements to affect the evaluating results, which are given by the researchers and experts. Researchers and experts marked the values in light of their experience, knowledge and the relative data of the timetable. So the deep-seated elements affecting timetable stability are the interior factors and the exterior factors, which are grade of the railway lines, blocking system type, station interlocking type, leading locomotive performance, average mileage of the sections, capacity load of the sections, capacity load of the stations, total time supplement for running in the sections, total time supplements for dwelling on the stations, timetable periodic time, buffer times among train paths.

Timetable stability is closely associated with the exterior timetable stability index $G^{\text {exterior }}$ and the interior timetable stability index $G^{\text {interior }}$. And we can see that the calculations of $G^{\text {exterior }}$ and $G^{\text {interior }}$ rely on Equation (4), which determines the computing results.

\section{Conclusion}

Factors which affect train timetable stability can be classified into two kinds, the interior and exterior. We can see that the information entropy theory is suitable for describing the interacting relations among the affecting factors of the train timetable stability. The normalization function hires the triangle function, which is feasible and easy to calculate. The interest of the proposed approach is to hire the entropy theory to evaluate the timetable stability, which cannot be seen in the previous publications. The method proposed can evaluate the train timetable stability effectively and can give some decision supporting information in timetable design process.

Future research is directed toward optimizing timetable stability based on the evaluating method with entropy theory to broaden the research field. And the goal is to optimize the timetable on the dispatching section as well as on the railway network. Moreover, it is important to consummate timetable evaluating and optimizing models, including more affecting factors, restrictions to get a more practical timetable with high quality.

\section{Acknowledgements}

This work is financially supported by the National Natural Science Foundation of China (Grant: 61263027), the State Key Laboratory of Rail Traffic Control and Safety (Contract No. RCS2011K004), Beijing Jiaotong University, the Fundamental Research Funds of Gansu Province (Grant: 620030) and New Teacher Project of Research Fund for the Doctoral Program of Higher Education of China (20126204120002). The authors wish to thank anonymous referees and the editor for their comments and suggestions. The authors would also like to thank Dr. Yong Qin and Jie Xu for their helpful comments on the earlier version of this paper. 


\section{References}

[1] Z. Yang, A. Hu, J. Li, K. Hu, Journal of the China Railway Society, 15, 46-56 (1993).

[2] L. Jia, Fuzzy control and decision and its application in atomization, Ph D Dissertation, China Academy of Railway Science.

[3] Q. Peng, S. Zhu, H. Yan, Journal of Southeast Jiaotong University, 33, 367- 371 (1998).

[4] J. Ma, H. Xu, S. Hu, Z. Chen, Journal of Northern Jiaotong University, 27, 46-50 (2003).

[5] Q. Peng, Y. Zhang, M. Li, Journal of Southeast Jiaotong University, 33, 372-377 (1998).

[6] P. Zhao, A. Hu, Journal of Northern Jiaotong University, 19 , 20-24 (1995).

[7] R. M. P Goverde, Proceedings of the International Conference on Computer Aided Design, Manufacture and Operation in The Railway and Other Advanced Mass Transit Systems, 6, 339-350 (1998).

[8] M. Carey, S. Carville, Journal of Operational Research Society, 51, 666-682 (2000).

[9] I. A. Hansen, Proceedings of Seventh International Conference on Computers in Railways, Computers in Railways, VII, 809-816 (2000).

[10] W. Zha, G. Xiong, System Engineering, 19, 47-51 (2001).

[11] A. F De Kort, B. Heidergott, H. Ayhan, European Journal of Operational Research, 148, 644-661 (2003).

[12] R. M. P Goverde, Transportation Research Part B, 41, 179201 (2007).

[13] J. Zong, Z. Li, Journal of Transportation Engineering and Information, 8, 56-61 (2010).

[14] J. Chen, X. Zhang, B. Xu, Journal of System Simulation, 23, 770-773 (2011).

[15] X. Meng, L. Jia, Y. Qin, Journal of Transportation Research Record, 2197, 71-79 (2010).

[16] O. Engelhardt-Funke, M. Kolonko, International Transactions in Operational Research, 11, 381-394 (2004).

[17] R. M. P. Goverde, Punctuality of railway operations and timetable stability analysis, PhD Dissertation, TRAIL Research School, Delft University of Technology.

[18] X. Delorme, X. Gandibleux, J. Rodriguez, European Journal of Operational Research, 195, 780-790 (2008).

[19] X. Meng, L. Jia, J. Xie, Y. Qin, Proceedings of Chinese Control and Decision Conference, 2533-2536 (2010).

[20] X. Meng, L. Jia, Y. Qin, J. Xu, Transactions of Beijing Institute of Technology, 32, 80-83 (2012).

[21] T. M Herrmann, Stability of timetable and train routings through station regions, Ph D Dissertation. ETH.

[22] J. Chen, Research on establishment and evaluation of the stability-based train working diagram of passenger dedicated lines, Master Dissertation, Beijing Jiaotong University.

[23] C. E. Shannon, ACM SIGMOBILE Mobile Computing and Communications Review, 5, 3-55 (2001).

[24] C. Bianca and V. Coscia, Applied Mathematics Letters, 24, 149-155 (2011).

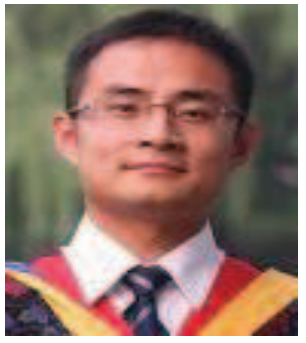

Xuelei Meng received the $\mathrm{PhD}$ degree in planning and management of traffic and transportation at State Key Laboratory of Rail Traffic Control and Safety, Beijing Jiaotong University in 2011. $\mathrm{He}$ is currently an associate professor in Lanzhou Jiaotong University. His research interests are in the areas of train timetable design, optimization and evaluation, line planning for railway. He has published research papers in reputed international journals, such as Transportation Research Record.

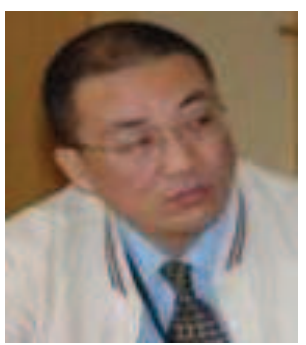

Limin Jia is a professor responsibility and doctoral supervisor of State Key Laboratory of Rail Traffic Control and Safety, Beijing Jiaotong University. $\mathrm{He}$ received his $\mathrm{PhD}$ degree at China Academy of Railway Science in 1991. He is the member of the Chinese national intelligent transportation expert advisory committee, China systems engineering society, China railway society, China operations research society. He is also an editorial member of China Railway Science. His main research interests are: application of intelligent control and intelligent systems, railway intelligent automation, railway intelligent transportation system. 